\title{
On the Effects of the Design of Cable-Driven Robots on Kinematics and Dynamics Models Accuracy
}

\author{
A. Gonzalez-Rodriguez ${ }^{a}$, F. J. Castillo-Garcia ${ }^{\text {b,* }}$, E. Ottaviano ${ }^{c}$, P. Rea ${ }^{c}$, A.G. \\ Gonzalez-Rodriguez ${ }^{\mathrm{d}}$ \\ a School of Industrial Engineering, University of Castilla-La Mancha. Av. Camilo Jose Cela \\ SN, 13071, Ciudad Real, Spain \\ ${ }^{b}$ School of Industrial Engineering, University of Castilla-La Mancha. Av. Carlos III SN, \\ 45071, Ciudad Real, Spain \\ ${ }^{c}$ DICeM, University of Cassino and Southern Lazio, Via G. Di Biasio 49, 03043 Cassino, \\ Italy \\ dDepartment of Electric Engineering and Automation, University of Jacn, Campus Las \\ Lagunillas SN,23071 Jaen, Spain
}

\begin{abstract}
This paper tackles the problem of improving the connection between the fixed frame and the end-effector of planar and spatial cable-driven robots. A new design concept is detailed, which consists in adding pulleys to the attachment between the cables and the end-effector. These reflective pulleys must have the same radius as the ones at the frame in order to compensate their geometry. Without this modification in the end-effector, the usual simplification of the point-to-point method used to model the connection between frame and endeffector leads to significant errors in the Kinematics and Dynamics of the system due to the fact that the geometry of the frame pulleys is disregarded. By adding the reflective pulleys in the end-effector, the equations of the Kinematics and Dynamics of the real system are equivalent to those of the point-to-point model, and therefore, this simplified method can be used without inherent errors. This solution may be of great importance for computational issues because it leads to codes that may run at real time. An analytical proof of the equivalence of both models is presented. Finally, experimental results have been conducted for
\end{abstract}

\footnotetext{
* Corresponding author

Email addresscs: antonio.gonzalez@uclm.es (A. Gonzalez-Rodriguez), fernando. castillo@uclm.es (F. J. Castillo-Garcia), ottaviano@unicas.it (E. Ottaviano), ottaviano@unicas.it (P. Rea), agaspar@ujaen.es (A.G. Gonzalez-Rodriguez)
} 
both, planar and spatial cable-robots, in order to illustrate the advantages of using this novel design concept for the connection between the end-effector and the fixed frame.

Keywords: cable-driven robot, kinematics model, dynamics model, positioning accuracy, end-effector attachment

\section{Introduction}

Cable-driven robots are a class of parallel manipulators [1] that consist of a moving end-effector connected to a fixed frame by means of cables $[2,3]$. The main components are the fixed frame (or structure), the end-effector, cables,

s. and the actuation and transmission system. A cable-based manipulator can operate the end-effector by changing the lengths of cables while preventing any cable of becoming slack.

During the last decade, several planar and spatial versions of cable-driven manipulators have been proposed $[4,5,6,7,8,9,10]$. In all these prototypes and design solutions, cables are commanded by electrical actuators, mainly DCmotors due to the necessary application of a torque control [11] to properly regulate the position/orientation of the end-effector [12]. The usual solution to connect DC-motors and end-effector by means of cables is to use winches that roll each cable in and out, as in [9]. They are attached at the motor shaft and 15 they require the so called guiding pulleys to change the direction of the cables and to transmit force [13]. While the guiding pulleys are used in all cases for guiding cables from the winch to the end-effector, in most of the works the cable attachments on the mobile platform is found in the form of cable attachment pegs, ideally represented by a single point $[3,4,7,8,9,10,12,13,14]$. Although on guiding pulleys are always used, few works describe their influence $[15,16]$, or reduce the positioning error by taking them into account in the kinematic model (e.g. [17]).

A second way to connect a cable at the fixed frame is the rail-based system in which cables are considered as links of constant length, driven by a skid-rail 
system. Although in most of proposed designs of this type [18] each two skids share a common rail, every skid is separately operated by a DC motor through a drive belt. This makes this design solution similar to a linear drive [19].

On the other hand, most of the published works present cable manipulator designs assuming that no sensor is available for a direct measurement of the end-effector position owing to the fact that this kind of sensors (which provide a precise real time measurement) are expensive [20]. Therefore, the most extended solution consists in estimating the end-effector pose by means of the position of the motor/gearbox or pulleys $[21,22]$ and/or the cables tensions $[23,16]$. Hence, a very accurate kinematic relation between end-effector position and 35 actuator position is needed to estimate the end-effector pose. Depending on the demanded accuracy, two kinds of approaches can be found:

a) Works in which the kinematic and dynamic effects of the pulleys are neglected and they are only taken into account to calculate the relation between the motor torque and the cable tension, and to estimate cables length variation $[24,25]$. In this case, these modeling assumptions may cause errors in the estimation of the end-effector pose (which is used as a reference in the control loop) and inherent errors will appear.

b) Works in which the kinematic effects of the pulley inclusion is assumed and included into the model $[26,17]$. In this case, the reference error described in a) is highly reduced, although the model complexity considerably increases.

It is less frequent to find works focused on improving mechanical designs in order to reach more accurate models (e.g. $[27,28]$ ). In this line, this paper introduces a new design concept that overcomes the problems described in a) and b), without increasing the complexity of such models. It consists in including pulleys in the end-effector that compensates the geometry of the pulleys in the frame. This way, the point-to-point model can be used to obtain an accurate relationship between end-effector pose and joints angles without introducing complex equations that take into account the non-punctual geometry of the 
pulleys/drums/winches in the frame. Preliminary results were presented in [29].

The remaining of the paper is organized as follows: Section 2 details the conventional modeling for cable robots and the Kinematics and Dynamics errors that originate. Section 3 presents the new design concept for the connection between frame and end-effector, which allows using the simplified models without affecting to Kinematics and Dynamics estimation. Section 4 supports the novel proposal by means of experimental results for both, a planar and a spatial case. Finally, Section 5 summarizes the main conclusions obtained in this work.

\section{Problem Statement}

\subsection{Conventional point-to-point model: spatial case}

This subsection is devoted to detail the conventional point-to-point model for spatial cable robots. This model assumes that cables connect the frame (from fixed points placed at pulleys centroid) to fixed points at the end-effector, neglecting the effects of the pulley radius.

In a first stage let's assume the planar $n$ cable robot of Fig.1, in which every cable go from a winch/drum attached to the actuator (Fig.1 detail 1), then to a pulley (detail 2), and, finally, to a fixed point at the end-effector (detail 3). The lengths of the cables are denoted by $L_{i}$, and the elevation angles by $\theta_{i}$. The rotation angle of each actuator is designed as $\alpha_{i}$. Positive values originate negative changes in the cables length, $\Delta L_{i}$, that can be expressed as $\Delta L_{i}=-\alpha_{i} r$, being $r$ the radius of the winches. Therefore, actuators angles can be obtained as

$$
\boldsymbol{\alpha}=-\frac{1}{r} \Delta \mathbf{L}
$$

In the case of a spatial robot (see Fig.2), the end-effector pose is defined as $\mathbf{Q}=\left[x, y, z, \varphi_{x}, \varphi_{y}, \varphi_{z}\right]^{T}$, being $\varphi_{x}, \varphi_{y}$ and $\varphi_{z}$ the Tait-Bryan angles used to express the end-effector orientation [30]. Cables connect the frame at $\left[x_{f i}, y_{f i}, z_{f i}\right]$ 80 to the end-effector at $\left[x_{e i}, y_{e i}, z_{e i}\right]$. In the same way, the orientation of cables is expressed in spherical coordinates by means of angles $\theta_{i}$ and $\phi_{i}$, where $\theta_{i}$ is 


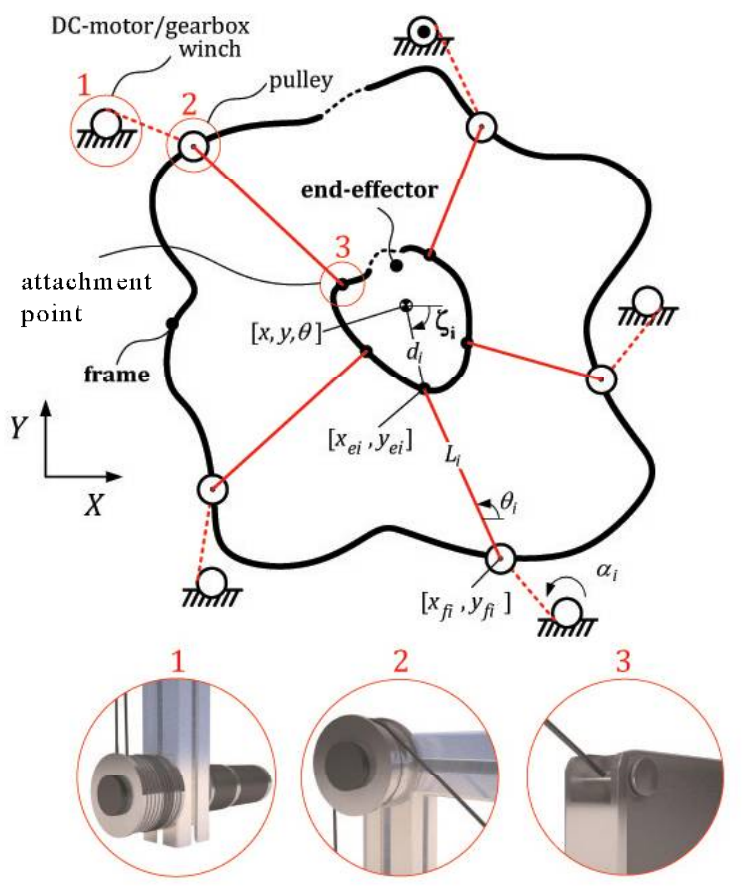

Figure 1: Conventional planar point-to-point model for $n$ cable-driven robot

the angle between the cable $i$ and its horizontal projection, and $\phi_{i}$ is the angle between this horizontal projection and the $x$ axis. In a similar way, and for the segment $i$ linking the end-effector centroid to the attachment point $i$, the angle $\zeta_{i}$ is the angle between this segment and the horizontal projection, and $\delta_{i}$ is the angle between this horizontal projection and the $x$ axis.

The length of each cable is

$$
\begin{aligned}
L_{i}= & {\left[\left(x_{f i}-x-d_{i} \cos \left(\zeta_{i}\right) \cos \left(\delta_{i}\right)\right)^{2}+\right.} \\
& \left(y_{f i}-y-d_{i} \cos \left(\zeta_{i}\right) \sin \left(\delta_{i}\right)\right)^{2}+ \\
& \left.\left(z_{f i}-z-d_{i} \sin \left(\zeta_{i}\right)\right)^{2}\right]^{\frac{1}{2}}
\end{aligned}
$$

which are controlled by modifying the set of actuator angles $\boldsymbol{\alpha}$ through (1). 


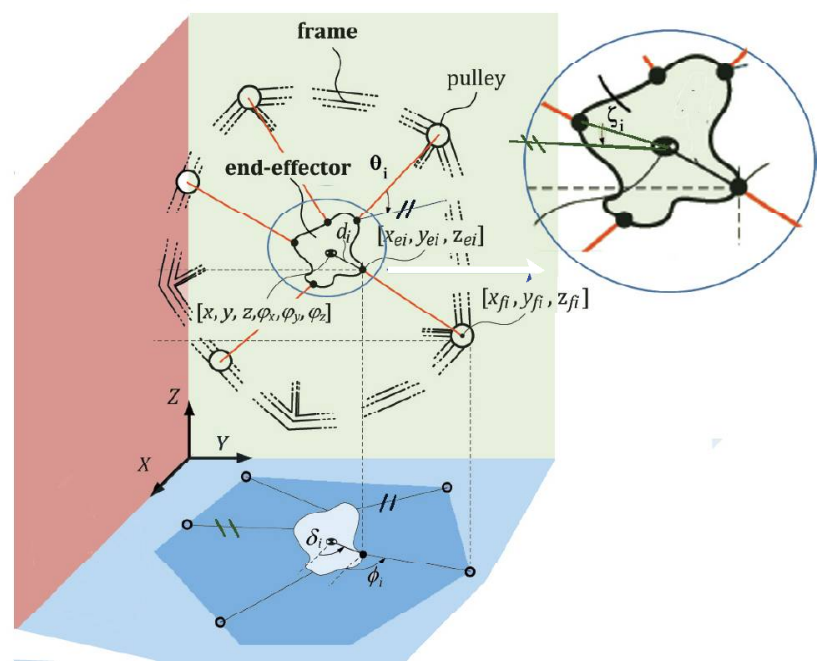

Figure 2: Spatial cable robot: frame/end-effector connection for point-to-point modeling.

In the planar case $\delta_{i}=0$. We can also use the $y$ coordinate as the vertical one

$$
\begin{aligned}
& L_{i}=\left[\left(x_{f i}-x-d_{i} \cos \left(\zeta_{i}\right)\right)^{2}+\left(z_{f i}-z-d_{i} \sin \left(\zeta_{i}\right)\right)^{2}\right]^{\frac{1}{2}} \rightarrow \\
& L_{i}=\left[\left(x_{f i}-x-d_{i} \cos \left(\zeta_{i}\right)\right)^{2}+\left(y_{f i}-y-d_{i} \sin \left(\zeta_{i}\right)\right)^{2}\right]^{\frac{1}{2}}
\end{aligned}
$$

It is worth noting that the angles $\zeta_{i}, \delta_{i}$ (only $\zeta_{i}$ in the planar case) can be obtained from the end-effector pose $\mathbf{Q}$ and the relative positions between the attachment points $\left[x_{f i}, y_{f i}, z_{f i}\right]$ and the centroid $[x, y, z]$. These positions only depends on the end-effector geometry, and hence they are constant. Once known these angles, the cable lengths can be obtained from (2), and finally, the actuator angles from (1). This procedure corresponds to the inverse Kinematics, which starts from the end-effector pose to obtain the actuator angles. It is denoted as $\boldsymbol{\alpha}=\Lambda^{I}(\mathbf{Q})$. The forward Kinematics starts from the actuator angles and obtain the end-effector pose. It is denoted as $\mathbf{Q}=\boldsymbol{\Lambda}^{F}(\boldsymbol{\alpha})$.

\subsubsection{Dynamics}

100 The end-effector dynamics can be expressed by 


$$
\mathbf{M Q}=\mathbf{F}
$$

where $\mathbf{M}$ is a diagonal matrix being $m_{11}=m_{22}=m_{33}=m$ (i.e. the end-effector mass), $m_{44}, m_{55}, m_{66}$ are the momenta of inertia along the respective axes, and $\mathbf{F}=\left[F_{x}, F_{y}, F_{z}, \tau_{x}, \tau_{y}, \tau_{z}\right]^{T}$, being $F_{x}, F_{y}$ and $F_{z}$ the Cartesian components of the force applied to the end-effector, and $\tau_{x}, \tau_{y}, \tau_{z}$ the respective torques.

On the other hand, $\mathbf{F}$ can be computed by means of the equilibrium of the end-effector forces and torque as

$$
\mathbf{F}=\mathbf{J}_{s} \mathbf{T}+\mathbf{F}_{g}
$$

where $\mathbf{F}_{g}=[0,0,-m g, 0,0,0]^{T}, \mathbf{T}=\left[T_{1}, \ldots, T_{n}\right]^{T}$, i.e. the array of cables tension, and $\mathbf{J}_{s}$ is the static Jacobian matrix, that yields

Finally, the static Jacobian matrix, $\mathbf{J}_{s}$ can be written as

$$
\mathbf{J}_{s}=\left[\begin{array}{cccc}
J_{11} & J_{12} & \ldots & J_{1 n} \\
J_{21} & J_{22} & \ldots & J_{2 n} \\
J_{31} & J_{32} & \ldots & J_{3 n} \\
J_{41} & J_{42} & \ldots & J_{4 n} \\
J_{51} & J_{52} & \ldots & J_{5 n} \\
J_{61} & J_{62} & \ldots & J_{6 n}
\end{array}\right]
$$

110 where

$$
\begin{aligned}
& J_{1 i}=\cos \left(\theta_{i}\right) \cos \left(\phi_{i}\right) \\
& J_{2 i}=\cos \left(\theta_{i}\right) \sin \left(\phi_{i}\right) \\
& J_{3 i}=\sin \left(\theta_{i}\right) \\
& J_{4 i}=\left(y_{e i}-y\right) \sin \left(\theta_{i}\right)-\left(z_{e i}-z\right) \cos \left(\theta_{i}\right) \sin \left(\phi_{i}\right) \\
& J_{5 i}=\left(z_{e i}-z\right) \cos \left(\theta_{i}\right) \cos \left(\phi_{i}\right)-\left(x_{e i}-x\right) \sin \left(\theta_{i}\right) \\
& J_{6 i}=\left(x_{e i}-x\right) \cos \left(\theta_{i}\right) \sin \left(\phi_{i}\right)-\left(y_{e i}-y\right) \cos \left(\theta_{i}\right) \cos \left(\phi_{i}\right)
\end{aligned}
$$

In the particular case of a planar robot, $\phi_{i}=0$ and the Jacobian matrix is 
reduced to a $3 \times 3$ one. It yields:

$$
\begin{aligned}
J_{1 i} & =\cos \left(\theta_{i}\right) \\
J_{2 i} & =\sin \left(\theta_{i}\right) \\
J_{3 i} & =\left(z_{e i}-z\right) \cos \left(\theta_{i}\right)-\left(x_{e i}-x\right) \sin \left(\theta_{i}\right)
\end{aligned}
$$

\subsection{Modeling errors}

It is well-known that providing a direct measurement of the end-effector pose, $\mathbf{Q}$, to be fedback in the control scheme, is expensive [20]. Therefore, most of the works assume that no sensors are available to provide $\mathbf{Q}$ by direct measurement. On the contrary, it is estimated from the forward Kinematics $\mathbf{Q}=\boldsymbol{\Lambda}^{F}(\boldsymbol{\alpha})$ and the actuator positions, $\boldsymbol{\alpha}$, which can be directly measured by means of conventional encoders placed at the motors or at the winches. As a consequence, accurate kinematic transformations are required in order to properly control the end-effector pose $[31,32]$.

As it was mentioned in Section 2.1, the conventional point-to-point model assumes that cables goes from the pulleys centroid, $\left[x_{f i}, y_{f i}, z_{f i}\right]$, to the attachment points at the end-effector, $\left[x_{e i}, y_{e i}, z_{e i}\right]$, although in a practical realization with pulleys in the frame, the cable ends at the exit point of this pulley $\left[x_{f i}^{r}, y_{f i}^{r}, z_{f i}^{r}\right]$. Figure 3 illustrates these modeling errors for a planar cable robot, in which superindex ${ }^{r}$ stands for the real variables. This assumption simplifies the model complexity but originates errors on cable lengths, $L_{i} \neq L_{i}^{r}$, and on cable angles, $\theta_{i} \neq \theta_{i}^{r}$. These deviations are not constant values since the position of the exit point of the cable from the frame pulley changes when the end-effector pose does. As a consequence, these deviations give rise to errors when formulating the Kinematics and Dynamics of the system.

As previously mentioned, some works modify the conventional modeling by including the effects of the frame pulleys of Fig. 3, but obviously the model complexity grows in comparison to the conventional models $[26,15]$. The following Section presents a novel mechanical design to at tach cables to end-effector which allows using the conventional models without getting into the mentioned Kinematics and Dynamics errors. 


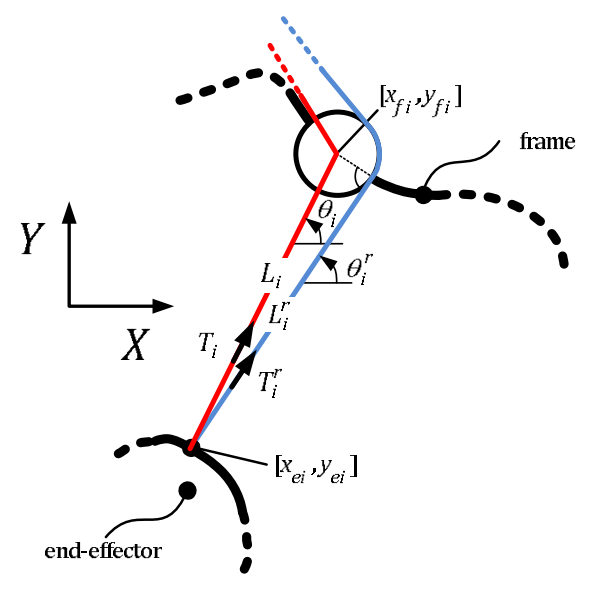

Figure 3: Deviation of the cable length and angle of the real configuration compared to the point-to-point model.

\section{The novel mechanical design solution}

A pulley (or also a drum or winch) located in the frame is required in almost all the cable-driven robot configurations. This non-punctual geometry originates a deviation with respect to the point-to-point model, which is usually preferred to model the drive. This section analyses the effect of adding a pulley in the end-effector, to compensate the pulley geometry in the frame. This modification must satisfy the following:

- The cables that connect the frame to the end-effector go from a pulley placed at a fixed point in the frame, to a second pulley placed at a point of the end-effector. Both pulleys have the same radius.

- The disposition between cable and pulleys responds to one of the first two situations shown in Fig. 4.

The kinematic equivalence for two robot configurations will be investigated: planar robots with two translational DoFs in a vertical plane; and spatial robots, with DoFs in the three Cartesian axes and a rotational DoF about the vertical 


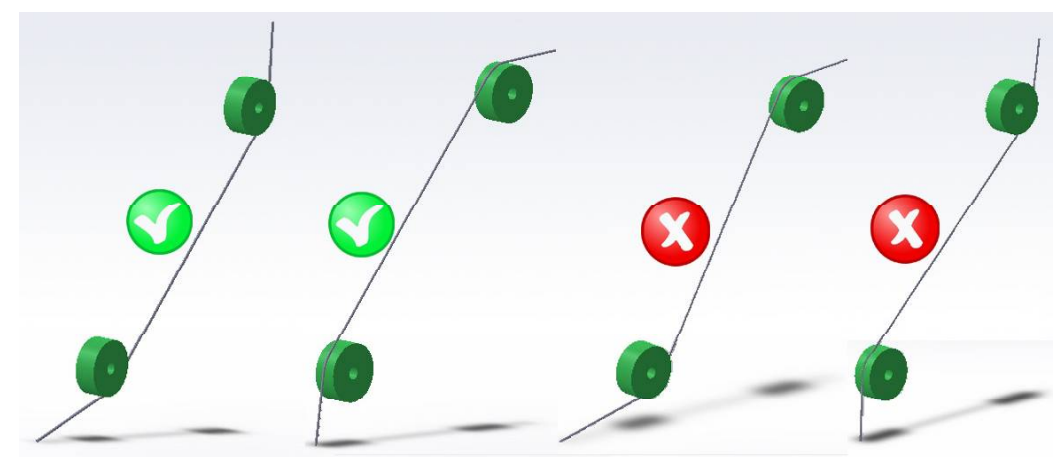

Figure 4: Situations in which a pulley in the end-effector compensates the errors originated by a pulley in the frame.

axis. In the third subsection the dynamic equivalence will be demonstrated for the more general spatial case.

\subsection{Planar robots}

In the following we demonstrate that, under certain conditions, the proposed modification, i.e. adding a fixed pulley at the end-effector, makes the physical system behaves as the point-to-point model detailed in the previous Section.

These conditions are:

- Only translations along the horizontal $(x)$ and/or vertical $(y)$ directions are allowed. Rotation is forbidden.

- At the end-effector, the relative position between the attachment point $\left[x_{a i}, y_{a i}\right]$ and the pulley is fixed.

This last condition implies that the cable exit points remain constant regardless of the end-effector position, and therefore (see Fig. 5) $\varepsilon_{i}^{A}, x_{e i}-x_{a i}$, and $y_{e i}-y_{a i}$ are constant values. In the frame, the relative position between winch and pulley is fixed, and therefore $L_{f r}$ and $\varepsilon_{i}^{D}$ are also constant.

In addition, this figure shows that $L_{i}=L_{i}^{r}$ and $\theta_{i}=\theta_{i}^{r}$, and also

$$
\varepsilon_{i}^{A}+\varepsilon_{i}^{B}=\pi-\varepsilon_{i}^{C}+\varepsilon_{i}^{D} \Rightarrow \varepsilon_{i}^{B}+\varepsilon_{i}^{C}=\text { constant }
$$




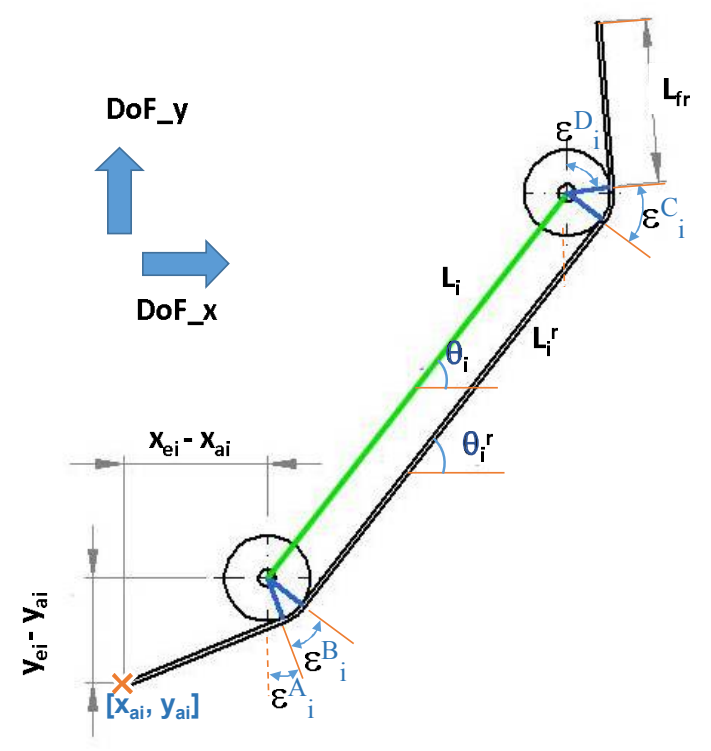

Figure 5: Fquivalence of angles and length in a planar configuration.

170 On the other hand, the total cable length $L_{i}^{r t}$ which can be measured through (1) consists of:

$$
L_{i}^{r t}=L_{e e}+\varepsilon_{i}^{B} r+L_{i}^{r}+\varepsilon_{i}^{C} r+L_{f r} \quad \text { with } L_{e e}=\sqrt{\left(x_{e i}-x_{a i}\right)^{2}+\left(y_{e i}-y_{a i}\right)^{2}}
$$

Since $L_{e e}, \varepsilon_{i}^{B}+\varepsilon_{i}^{C}$, and $L_{f r}$ are constant, and also $L_{i}=L_{i}^{r}$, then from the geometrical point of view, the value of $L_{i}$ required to operate in the pointto-point model can be obtained in a straightforward way by measuring the 175 total cable length from (1) and applying (10). Therefore, including a fixed pulley in the end-effector allows using the equations for the inverse and forward kinematic transformations obtained for the point-to-point model without the errors illustrated in Fig.3. In addition, $\theta_{i}=\theta_{i}^{r}$.

\subsection{Spatial robots}

In an analogous way that in the case of planar robots, and under similar conditions, the real system behavior and the point-to-point model detailed in 
the previous Section are equivalent. These conditions are

- Translations are allowed along the three Cartesian axes. Orientation of the end-effector is allowed about the vertical axis (see Fig. 6a).

- The pulley at the end-effector can rotate $(\phi)$ about its vertical axis to accommodate the connection between pulleys when the end-effector moves in the horizontal plane, or it rotates about the vertical axis.

- At the end-effector, the attachment point must belong to the axis of orientation of the pulley (as in Fig. 6a).

Figure 6b) compares the point-to-point model scheme to the proposed one.

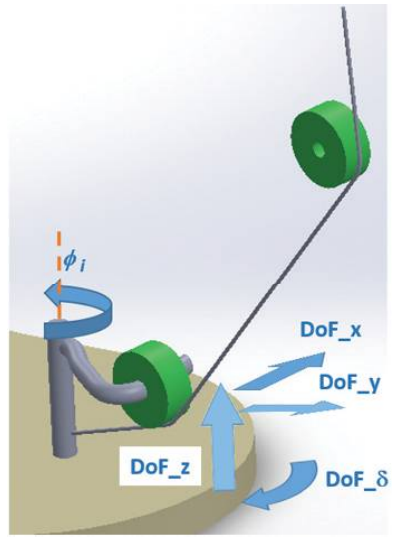

(a)

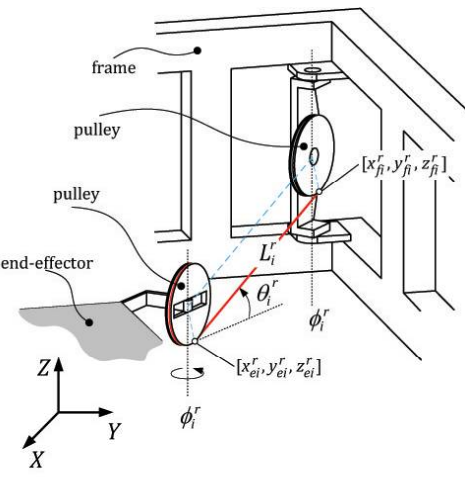

(b)

Figure 6: Spatial point-to-point scheme (solid red) and the proposed scheme (dashed blue)

In the spatial case, the reflective pulley is allowed to orientate such that the following items are all in the same vertical plane:

- in the end-effector: the attachment point, the pulley center, and the exit point $\left[x_{e i}^{r}, y_{e i}^{r}, z_{e i}^{r}\right]$

- the cable

- in the frame: the exit point $\left[x_{f i}^{r}, y_{f i}^{r}, z_{f i}^{r}\right]$, and the pulley center 
Since all of the involved elements are in the same plane, the previous reasoning for planar robots is valid for spatial ones. This way, adding reflective pulleys in the end-effector guarantees that the lengths $L_{i}^{r}$ are the same as in the point-to-point model, $L_{i}$. This means that the inverse and forward kinematics transformations $\Lambda^{\prime}$ and $\boldsymbol{\Lambda}^{\prime}$ are equivalent to the ones obtained in the previous Section for the point-to-point model. The cables lengths $L_{i}^{r}$ can be obtained from the motor angle through (1) and (10). In addition, the angles satisfy $\theta_{i}^{r}=\theta_{i}$ and $\phi_{i}^{r}=\phi_{i}$.

\subsection{Equivalence of the Dynamics}

In the more general case of spatial configurations, the static Jacobian matrix, $\mathbf{J}_{\mathbf{s}}^{\mathbf{r}}$, can be written as $(6,7)$, in which the point-to-point variables must be changed into real variables (with superindex ${ }^{r}$ ), resulting in

$$
\begin{aligned}
J_{1 i}^{r} & =\cos \left(\theta_{i}^{r}\right) \sin \left(\phi_{i}^{r}\right) \\
J_{i 2}^{r} & =\cos \left(\theta_{i}^{r}\right) \cos \left(\phi_{i}^{r}\right) \\
J_{i 3}^{r} & =\sin \left(\theta_{i}^{r}\right) \\
J_{4 i}^{r} & =\left(y_{e i}^{r}-y\right) \sin \left(\theta_{i}^{r}\right)-\left(z_{e i}^{r}-z\right) \cos \left(\theta_{i}^{r}\right) \sin \left(\phi_{i}^{r}\right) \\
J_{5 i}^{r} & =\left(z_{e i}^{r}-z\right) \cos \left(\theta_{i}^{r}\right) \cos \left(\phi_{i}^{r}\right)-\left(x_{e i}^{r}-x\right) \sin \left(\theta_{i}^{r}\right) \\
J_{6 i}^{r} & =\left(x_{e i}^{r}-x\right) \cos \left(\theta_{i}^{r}\right) \sin \left(\phi_{i}^{r}\right)-\left(y_{e i}^{r}-y\right) \cos \left(\theta_{i}^{r}\right) \cos \left(\phi_{i}^{r}\right)
\end{aligned}
$$

The exit points in the end-effector pulley result (see Fig. 6)

$$
\left[\begin{array}{c}
x_{e i}^{r} \\
y_{e i}^{r} \\
z_{e i}^{r}
\end{array}\right]=\left[\begin{array}{c}
x_{e i}+r \cos \left(\theta_{i}^{r}\right) \cos \left(\phi_{i}^{r}\right) \\
y_{e i}+r \cos \left(\theta_{i}^{r}\right) \sin \left(\phi_{i}^{r}\right) \\
z_{e i}+r \cos \left(\theta_{i}^{r}\right)
\end{array}\right]
$$




$$
\begin{aligned}
& J_{1 i}^{r}=\cos \left(\theta_{i}^{r}\right) \sin \left(\phi_{i}^{r}\right) \\
& J_{i 2}^{r}=\cos \left(\theta_{i}^{r}\right) \cos \left(\phi_{i}^{r}\right) \\
& J_{i 3}^{r}=\sin \left(\theta_{i}^{r}\right) \\
& J_{4 i}^{r}=\left(y_{e i}-y\right) \sin \left(\theta_{i}^{r}\right)-\left(z_{e i}-z\right) \cos \left(\theta_{i}^{r}\right) \sin \left(\phi_{i}^{r}\right) \\
& J_{5 i}^{r}=\left(z_{e i}-z\right) \cos \left(\theta_{i}^{r}\right) \cos \left(\phi_{i}^{r}\right)-\left(x_{e i}-x\right) \sin \left(\theta_{i}^{r}\right) \\
& J_{6 i}^{r}=\left(x_{e i}-x\right) \cos \left(\theta_{i}^{r}\right) \sin \left(\phi_{i}^{r}\right)-\left(y_{e i}-y\right) \cos \left(\theta_{i}^{r}\right) \cos \left(\phi_{i}^{r}\right)
\end{aligned}
$$

that are equals to the point-to-point model ones due to $\theta_{i}^{r}=\theta_{i}$ and $\phi_{i}^{r}=\phi_{i}$.

As it has been demonstrated, inverse and forward kinematics transformations, $\Lambda^{I}$ and $\Lambda^{I}$ are equivalent to the point-to-point model ones, and static Jacobian matrix, $\mathbf{J}_{s}$, is also equivalent. Therefore, the point-to-point Dynamics model can be also accurately used for the new proposed spatial cable robot using pulleys at the end-effector.

\subsection{Effects of the reflective pulley on the Dynamics}

Pulleys in the frame of cable-driven parallel robot are necessary in their practical realization to guide cables from winches to the end-effector, but they introduce modifications in the applied force due to the presence of friction and inertia. Modeling and compensation of friction effects in cable-driven parallel robot design are proposed in [16], in which Coulomb and Dahl models are derived and used to compensate errors in cable forces measurements. The inertia effect is discussed in [33]. The case of the reflective pulley in the end-effector as proposed in this paper is a different issue. In the planar case, it is fixed since it does not roll or rotate. In fact, it can be substituted by a circular shape where the cable lay on, as will be seen in Section 4. Therefore, inertia and friction are not of consideration.

With regard to the spatial configuration, variations in the fourth DoF (the end-effector orientation $\delta$ of Fig. 5) leads to variations (with opposite sign) in $\phi_{i}$. This angle also varies when the end-effector translates in the horizontal plane. It is worth noting that this pulley does not roll, and only the rotation 
about the vertical axis is allowed. Due to the small weight of the rotating part and the fact that this rotation $\dot{\phi}_{i}$ is slow, the effect of its inertia or friction is very reduced. Nevertheless, a complete study of these effects should be deeply performed in the Dynamics analysis in order to estimate the loss of accuracy that they originate, although it is out of the scope of this analysis.

\section{Experimental results}

This section presents the experiments to obtain the positioning error made when using the new proposal to connect the frame to the end-effector. The first subsection presents the experimental platform in which planar and spatial experiments have been conducted. The second and the third subsections present the planar and the spatial results, respectively.

\subsection{Experimental test-bed}

The prototype has been designed with Solidworks( (R) and built with aluminium profiles and 3D printed plastic parts $(P L A)$. The final design and prototype are shown in Fig. 7.

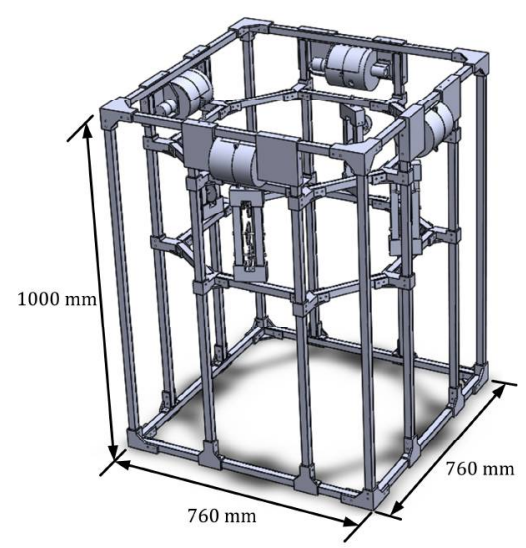

(a.)

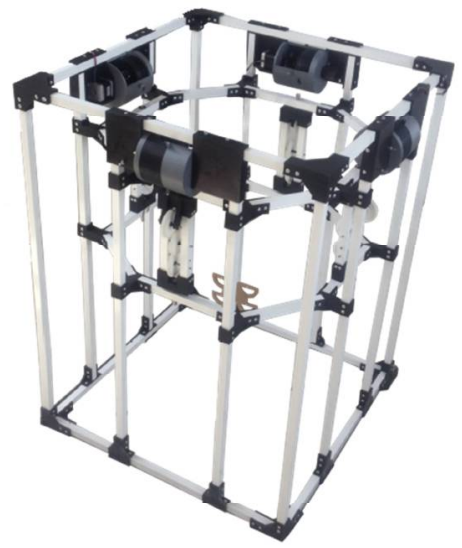

(b)

Figure 7: Fxperimental platform: a) Solidworks design; b) real prototype 
The overall dimensions of the prototypes are $760 \times 760 \times 1000 \mathrm{~mm}$. In the frame, four stepper motors (NEMA 17), move four mechanisms which allow the cables to be rolled in and out on the drums. This roller mechanism makes that the exit point of cables remains invariant when the drum rotates (see Fig. 8a). Figures $8 \mathrm{~b}$ and $8 \mathrm{c}$ show the actuator design and the final aspect of the roller mechanism. It is worth noting that each mechanism rolls a pair of cables.

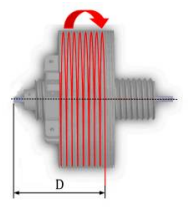

(a)

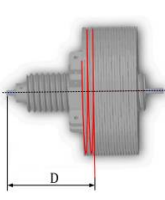

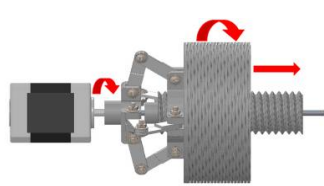

(b)

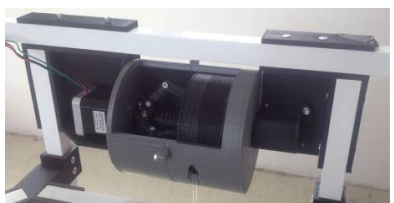

(c)

Figure 8: Roller mechanism: a) fixed location of the exit point; b) actuation design; c) real actuator

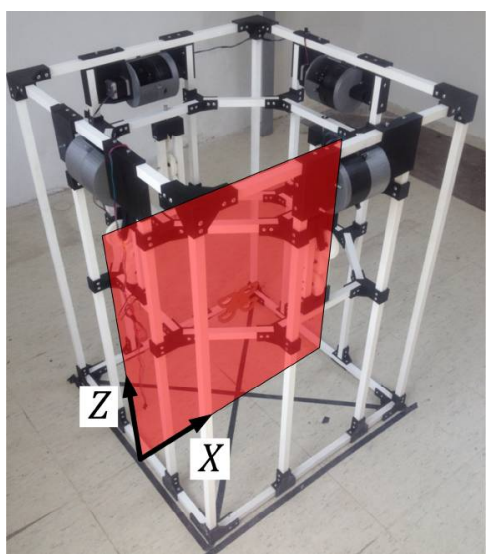

(a)

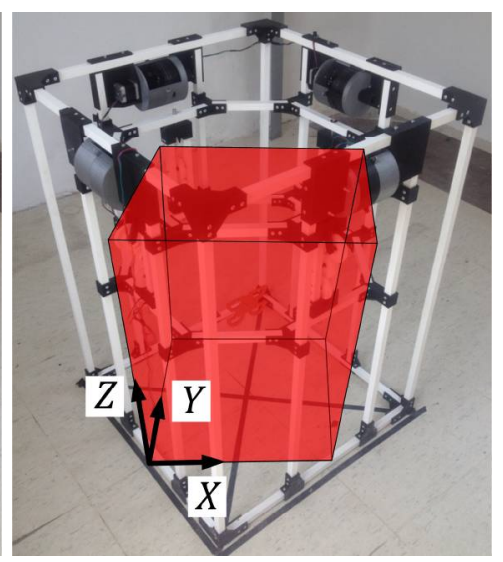

(b)

Figure 9: a) Planar (two motors) and b) spatial (four motors) configurations

The designed manipulator can be configured to be used as a planar one (two motors which two pairs of cables), or as an spatial one (four motors with four pairs of cables). In the planar case, the end-effector has two degrees-of-freedom, which are the translations in a vertical plane. The spatial case has four degrees- 
of-freedom, allowing the end-effector positioning, and the rotation about the vertical axis; the rotations in the $x$ and $y$ axes are blocked. Figure 9 details both configurations. The workspace of each robot (see Fig. 10) can be obtained assuming an end-effector pose and checking if all cable tensions remains positive (see $[10]$ for more details).

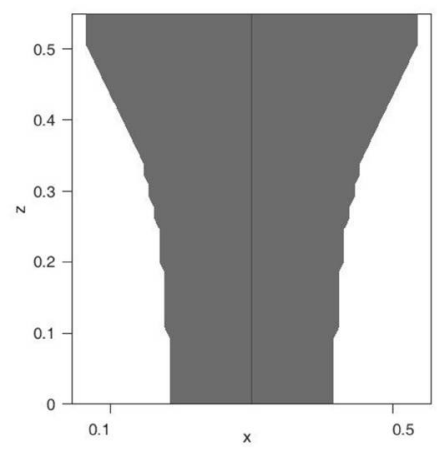

(a)

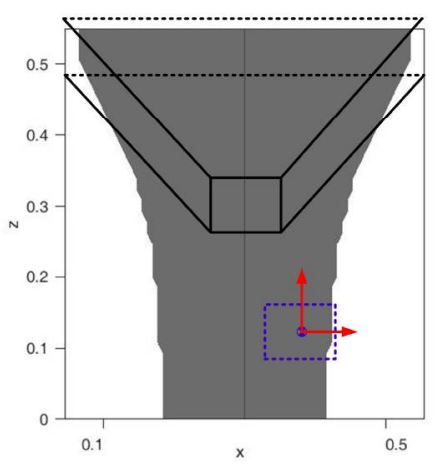

(b)

Figure 10: Workspaces for a) planar and b) spatial configuration. Values are given in $\mathrm{m}$

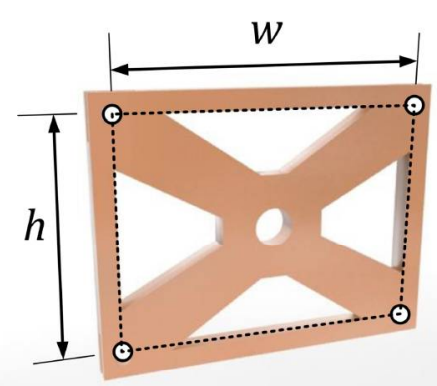

(a)

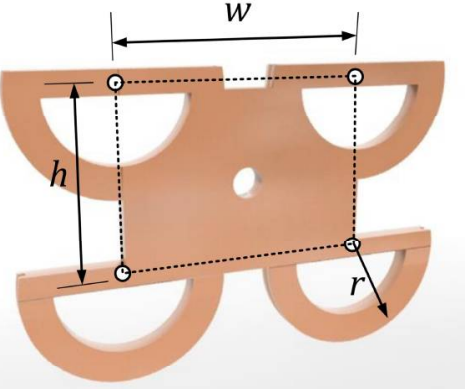

(b)

Figure 11: Compared end-effectors: a) without pulleys and b) with fixed pulleys

In the planar configuration, a more complete experiment has been conducted in order to verify the reduction of the positioning error if fixed pulleys are included at the end-effector connection. To this end, two different end-effectors 
have been compared: without pulleys as in the traditional case, and with fixed pulleys. Figure 11 shows both end-effectors.

With regard to the spatial configuration, only the proposed modification has been tested. The end-effector is shown in Fig. 12. with the pulleys rotating about vertical axes.

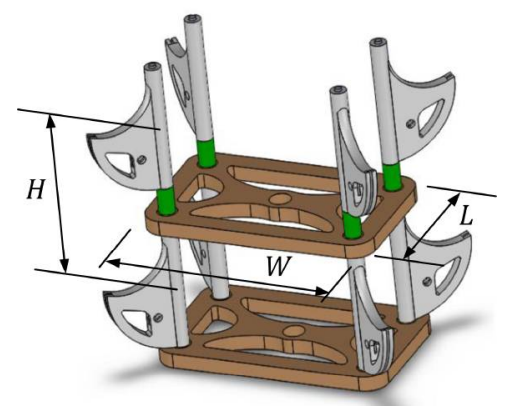

Figure 12: Spatial manipulator: end-effector

The main parameters of the robot are summarised in Table 1, specifying the differences between both experiments.

\subsection{Case 1: Two DoF planar cable robot}

\subsubsection{Trajectories}

This subsection compares the accuracy of the point-to-point Kinematics model using, on the one hand, a conventional punctual attachment in the endeffector (Fig. 11a) and, on the other, including a pulley in the end-effector side (Fig. 11b). To this aim, two planar trajectories have been tested for end-effector positioning: a circle (100 mm of diameter) and a rhombus (50 mm of side).

The time profile of both trajectories are a 4th order Bezier one to ensure the differentiability of its velocity and acceleration. The centre of both trajectories has been placed at the centre of the workspace and are $10 \mathrm{~s}$ duration.

Figures 13 and 14 respectively shows the sequence of movements of the circle trajectory and the rhombus one, with and without pulleys. 
Table 1: Parameters of the manipulator with planar configuration

\begin{tabular}{clr} 
Symbol & Description & Value \\
\hline & & \\
$H$ & height of the frame & $600 \mathrm{~mm}$ \\
$W$ & width of the frame & $566 \mathrm{~mm}$ \\
$L$ & length of the frame & $566 \mathrm{~mm}$ \\
$h$ & height of the end-effector & $60 \mathrm{~mm}$ \\
$w$ & width of the end-effector (planar) & $80 \mathrm{~mm}$ \\
$w$ & width of the end-effector (spatial) & $90 \mathrm{~mm}$ \\
$l$ & length of the end-effector (spatial) & $45 \mathrm{~mm}$ \\
$m$ & mass of the end-effector (planar) & $112 \mathrm{~g}$ \\
$m$ & mass of the end-effector (spatial) & $248 \mathrm{~g}$ \\
$r$ & radius of pulleys (end-effector) & $30 \mathrm{~mm}$ \\
$r$ & radius of drums/winches (frame) & $30 \mathrm{~mm}$
\end{tabular}

The accuracy of our robot is estimated by means of vision techniques. Computer vision cannot be used to feedback the real end-effector position (as occurs in conventional serial robot), but it is a suitable technique to measure the tracking error. MATLAB Image Toolbox (1) has been used to obtain an image binarization, compensate the tangential error and compare the real trajectory to the reference for both cases. By overlapping the reference and the real trajectory, it is possible to measure the perpendicular distance between them and proceed to directly obtain the error.

Due to the resolution of the camera, each image pixel corresponds to 0.085 mm approximately.

By defining the error in the normal direction to the reference trajectory, it is possible to obtain the number of pixels from the desired reference to the real trajectory and therefore obtain the tracking error. The compared results are shown in Fig. 15. 


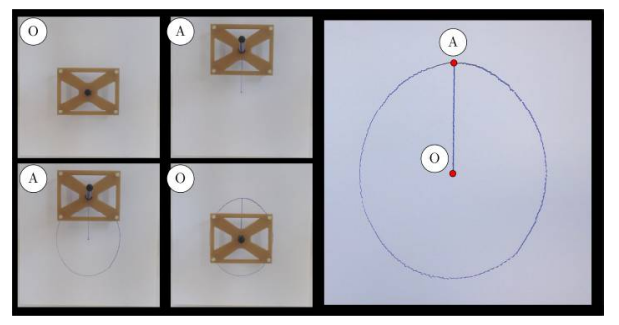

(a)

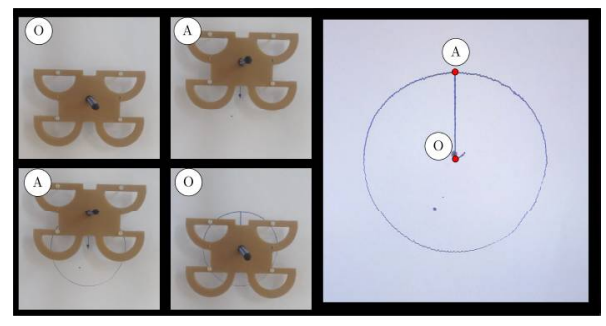

(b)

Figure 13: Front view of the circle trajectory on a vertical plane: a) without pulleys and b) with fixed pulleys

The results show that with the conventional point attachment, the maximum tracking error is $6.89 \mathrm{~mm}$ (and the average one is $2.24 \mathrm{~mm}$ ), whereas with the proposed pulley attachment, the maximum tracking error is $1.26 \mathrm{~mm}$ (and the average one is $0.28 \mathrm{~mm}$ ).

Finally, the same procedure has been followed for the rhombus trajectory.

305 Figure 16 represents the error for both compared attachment methods.

The results show again a significant reduction in the positioning error in comparison to the conventional point attachment. The maximum tracking error for the conventional point attachment is $9.00 \mathrm{~mm}$ (and the average one is 2.01 $\mathrm{mm})$. With the proposed pulley attachment, the maximum tracking error is $0.76 \mathrm{~mm}$ (and the average one is $0.17 \mathrm{~mm}$ ).

Therefore, adding fixed pulleys to the end-effector (with the same radius as the frame pulleys) significantly decreases the positioning error with regards to the conventional point connection to the end-effector. It is worth noting that 


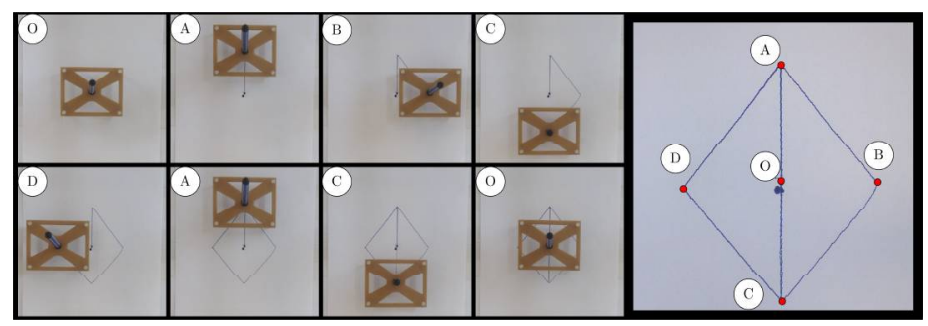

(a.)

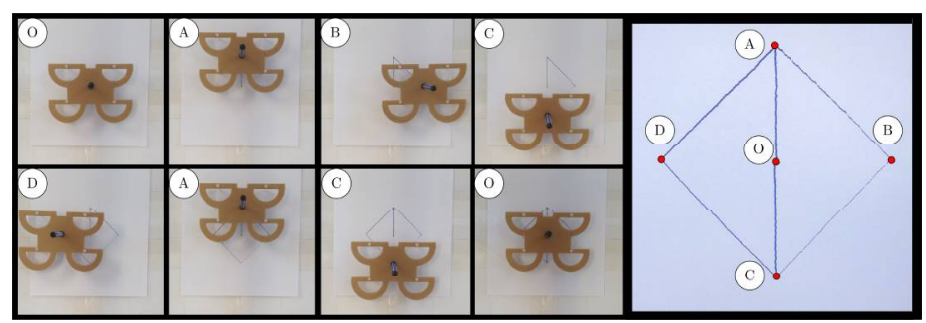

(b)

Figure 14: Front view of the rhombus trajectory on a vertical plane: a) without pulleys and b) with fixed pulleys
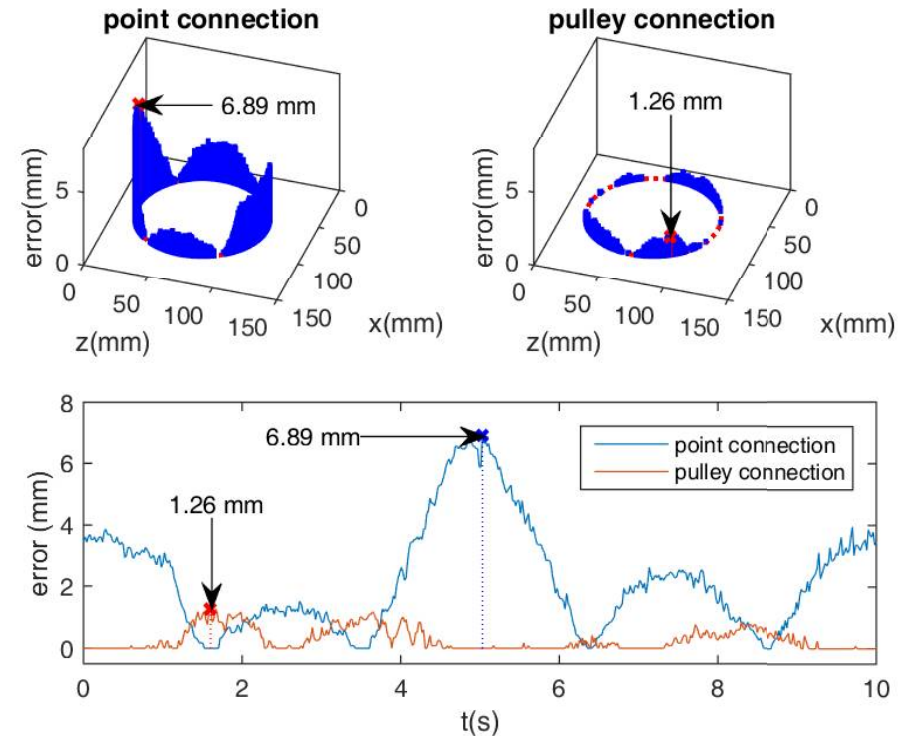

Figure 15: Circle trajectory comparison: tracking error 

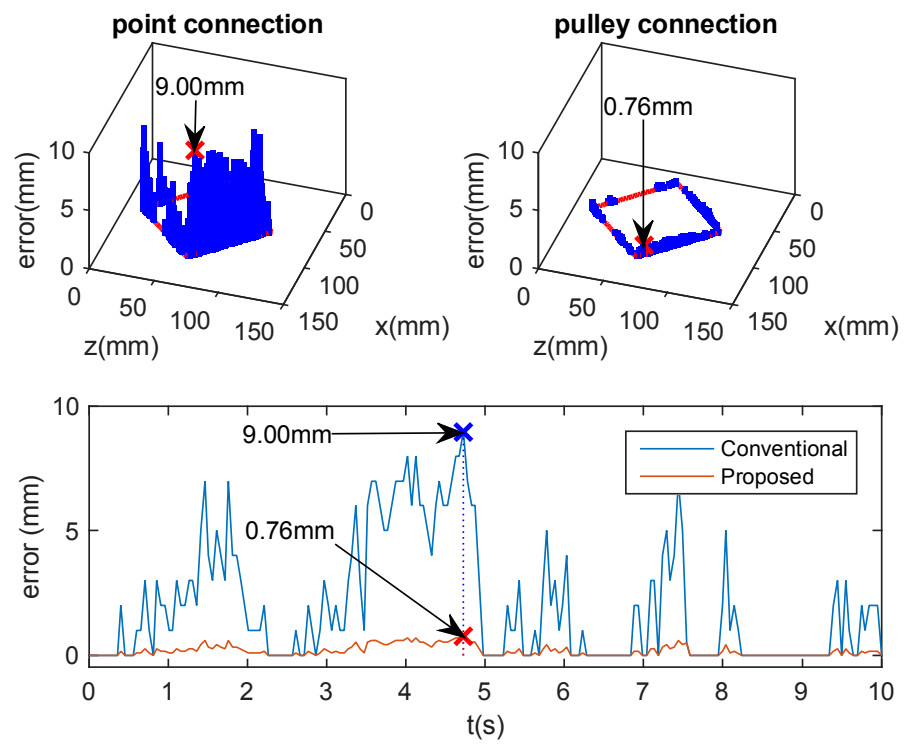

Figure 16: Rhombus trajectory comparison: tracking error

the error made using the conventional point attachment at the end-effector can be also reduced by software compensation (e.g. [32]) or taking into account the radius of the pulleys in the kinematic model (e.g. $[34,17])$, but the proposed solution provides an easier way to use the conventional point-to-point kinematic model without the error obtained when the frame pulleys radius is not taken into account $[13]$.

\subsection{Case 2: Four Dof spatial cable robot}

\subsubsection{Trajectories}

In this case, only the new pulley connection will be checked. The trajectories are a circumference $(150 \mathrm{~mm}$ of diameter) and a rhombus ( $75 \mathrm{~mm}$ of side), both executed into the $X Y$ plane.

The time profile of both trajectories are again a 4th order Bezier. The center of both trajectories has been placed at the center of the workspace and are $10 \mathrm{~s}$ duration. 


\subsubsection{Results}

Using an analogous procedure of the previous subsection (MATLAB Image Toolbox $(\mathrm{B})$ ), the error of both trajectories has been obtained. They are shown in Fig. 17 and 18.
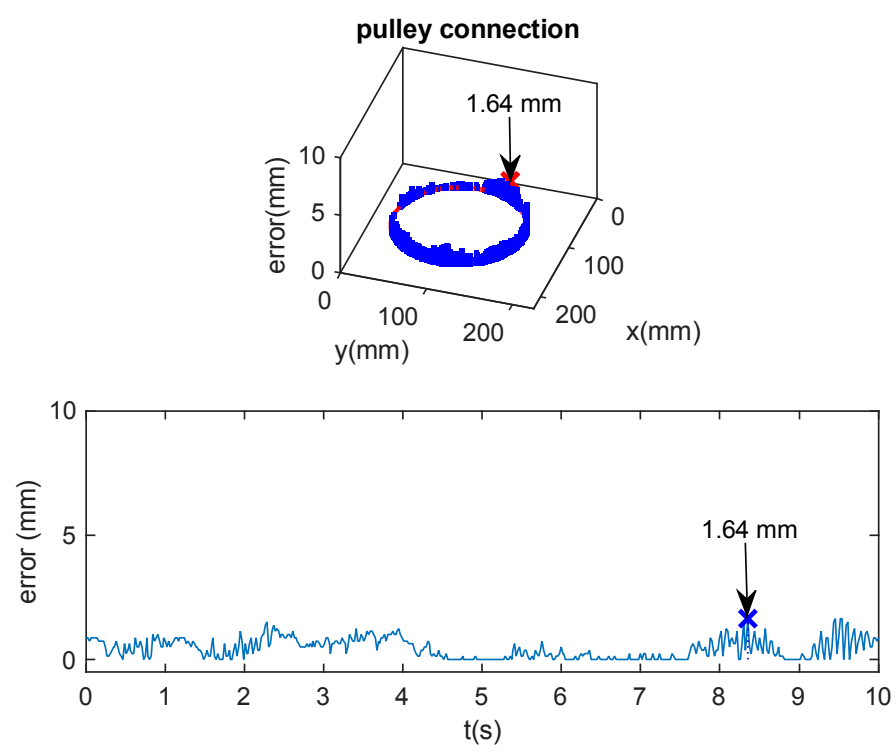

Figure 17: Circle trajectory error

As in the planar case, the results show that the proposed frame/end-effector connection gives rise to low values of positioning errors. For the circle trajectory, the maximum tracking error is $1.64 \mathrm{~mm}$ and its average one is $0.44 \mathrm{~mm}$. For a rhombus trajectory, the maximum tracking error for the conventional point connection is $1.01 \mathrm{~mm}$ and its average one is $0.46 \mathrm{~mm}$.

\section{Conclusions and future works}

In this paper, a new design solution is proposed to connect the fixed frame to the end-effector in cable-driven robots.

The conventional point-to-point model to describe the kinematic and dynamic behavior of cable robots has been recall for both planar and spatial cables 

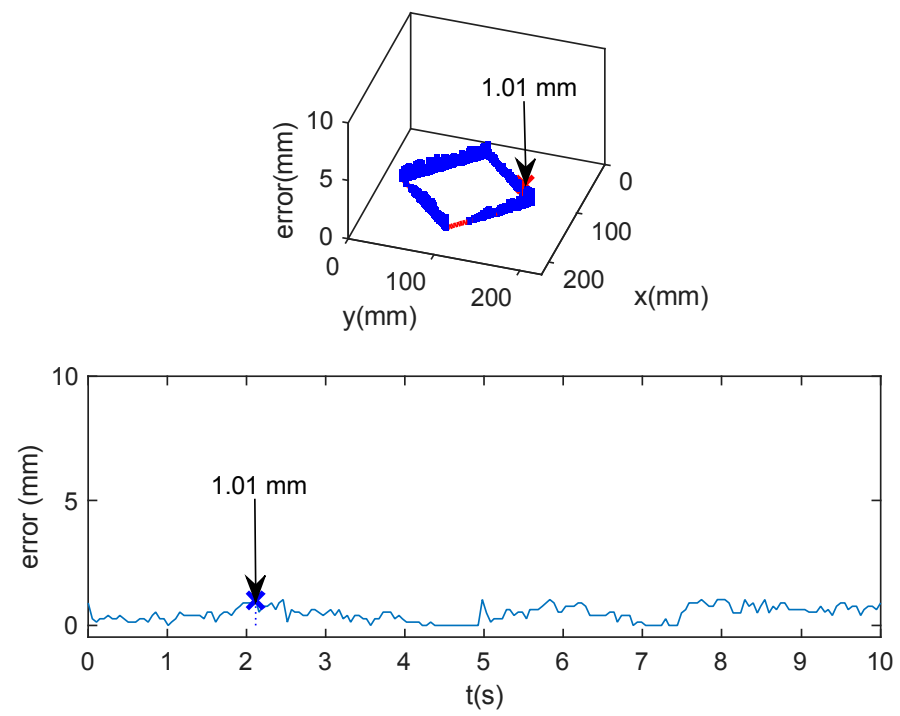

Figure 18: Rhombus trajectory error

robots. This usual way of modeling assumes that no pulleys are placed at the fixed frame, but the radius is only taken into account to compute cables lengths variations when drums roll in or out them. Indeed, it is shown in this paper that important inherent errors appears when this simplification is made due to the difference between the lengths and orientations of the cables in the model and the real system, which in turn originate deviations in the robot Kinematics and Dynamics.

In order to overcome the mentioned problems, we propose a novel design solution that includes pulleys at the end-effector, with the same radius as the fixed pulleys or winches at the frame. This solution makes the cable lengths and orientations equivalent to those of the point-to-point model. In addition, the static Jacobian matrix is also analytically equivalent to the point-to-point model which can then be used to describe the system dynamic behavior without 355 the mentioned errors.

Finally, two application examples that support the proposal have been pre- 
sented: a two DoF cable manipulator and a four DoF spatial one. In both cases, using the point-to-point conventional model with the proposed mechanical modification, the maximum positioning error is about $1 \mathrm{~mm}$.

As the theoretical analysis and experiments show, adding fixed pulleys to the end-effector allows using the conventional and simple point-to-point kinematic model while avoiding the inaccuracy derived from disregarding the pulleys radius (as detailed in [13]). This proposal can be considered an alternative way to the actual techniques consisting in software compensation (e.g. [32]) or the inclusion of the radius of the pulleys in the kinematic model (e.g. $[34,17])$

In its present form, the proposed modification of the attachment between cable and end-effector is not of application in all of the cable-driven robot configurations, but it is valid in the very typical configuration of 3-positioning DoFs with vertical orientation DoF, which is the preferred configuration in many ap370 plications (e.g. storing or palletization).

\section{References}

[1] J.-P. Merlet, Parallel robots, Vol. 74, Springer Science \& Business Media, 2012.

[2] R. Verhoeven, Analysis of the workspace of tendon-based stewart platforms, Ph.D. thesis, Universität Duisburg-Essen, Fakultät für Ingenieurwissenschaften Maschinenbau und Verfahrenstechnik (2004).

[3] R. G. Roberts, T. Graham, T. Lippitt, On the inverse kinematics, statics, and fault tolerance of cable-suspended robots, Journal of Robotic Systems $15(10)(1998) 581-597$.

380 [4] R. Babaghasabha, M. A. Khosravi, II. D. Taghirad, Adaptive robust control of fully-constrained cable driven parallel robots, Mechatronics 25 (2015) 27 36. doi:http://dx.doi.org/10.1016/j.mechatronics.2014.11.005.

[5] M. A. Khosravi, H. D. Taghirad, Robust $\{$ PID $\}$ control of fully-constrained 
cable driven parallel robots, Mechatronics 24 (2) (2014) 87-97. doi:http:

[6] L. Tang, X. Tang, X. Jiang, C. Gosselin, Dynamic trajectory planning study of planar two-dof redundantly actuated cable-suspended parallel robots, Mechatronics 30 (2015) 187-197. doi:http://dx.doi.org/10.1016/j. mechatronics.2015.07.005.

[7] R. Babaghasabha, M. A. Khosravi, H. D. Taghirad, Adaptive Control of KNTU Planar Cable-Driven Parallel Robot with Uncertainties in Dynamic and Kinematic Parameters, Springer International Publishing, Cham, 2015, pp. 145-159. doi:10.1007/978-3-319-09489-2_11.

[8] X. Jin, D. I. Jun, X. Jin, J. Seon, A. Pott, S. Park, J.-O. Park, S. Y. Ko, Upper Limb Rehabilitation Using a Planar Cable-Driven Parallel Robot with Various Rehabilitation Strategies, Springer International Publishing, Cham, 2015, pp. 307-321. doi:10.1007/978-3-319-09489-2_22.

[9] D. Nguyen, M. Gouttefarde, Study of reconfigurable suspended cabledriven parallel robots for airplane maintenance, in: 2014 IEEE/RSJ International Conference on Intelligent Robots and Systems, 2014, pp. 16821689. doi:10.1109/IROS.2014.6942781.

[10] G. Castelli, E. Ottaviano, A. González, Analysis and simulation of a new cartesian cable-suspended robot, Proceedings of the Institution of Mechanical Engineers, Part C: Journal of Mechanical Engineering Science 224 (8) (2010) $1717-1726$.

[11] V. Feliu, F. J. Castillo, F. Ramos, J. A. Somolinos, Robust tip trajectory tracking of a very lightweight single-link flexible arm in presence of large payload changes, Mechatronics 22 (5) (2012) 594613 .

[12] C. Gosselin, M. Grenier, On the determination of the force distribution inoverconstrained cable-driven parallel mechanisms, Meccanica 46 (1) (2011) 3-15. doi: $10.1007 / \mathrm{s} 11012-010-9369-\mathrm{x}$. 
[13] A. Pott, Influence of Pulley Kinematics on Cable-Driven Parallel Robots, Springer Netherlands, Dordrecht, 2012, pp. 197-204. doi:10.1007/ 978-94-007-4620-6_25.

[14] X. Diao, O. Ma, Vibration analysis of cable-driven parallel manipulators, Multibody system dynamies 21 (4) (2009) 347-360.

[15] L. Notash, D. McColl, Workspace investigation of wire-actuated parallel manipulators with uncertainties in wire connections, in: ASME 2010 International Design Engineering Technical Conferences and Computers and Information in Engineering Conference, American Society of Mechanical Engineers, 2010, pp. 213-222.

[16] W. Kraus, M. Kessler, A. Pott, Pulley friction compensation for winchintegrated cable force measurement and verification on a cable-driven parallel robot, in: 2015 IEEE International Conference on Robotics and Automation (ICRA), 2015, pp. 1627-1632. doi:10.1109/ICRA. 2015.7139406.

[17] M. Gouttefarde, D. Q. Nguyen, C. Baradat, Kinetostatic analysis of cabledriven parallel robots with consideration of sagging and pulleys, in: Advances in Robot Kinematics, Springer, 2014, pp. 213221.

[18] J.-P. Merlet, Kinematics of the wire-driven parallel robot marionet using linear actuators, in: Robotics and Automation, 2008. ICRA 2008. IEEE International Conference on, IEEE, 2008, pp. 3857-3862.

[19] J.-P. Merlet, Marionet, a family of modular wire-driven parallel robots, in: Advances in Robot Kinematics: Motion in Man and Machine, Springer, 2010, pp. 53-61.

[20] II. Kino, S. Yabe, C. Cheah, S. Kawamura, S. Arimoto, A motion control scheme in task oriented coordinates and its robustness for parallel wire driven systems, Journal-Robotics Society of Japan 18 (3) (2000) 103-110.

[21] A. Lytle, F. Proctor, K. Saidi, Control of Cable Robots for Construction Applications, INTECII Open Access Publisher, 2008. 
[22] P. Gholami, M. M. Aref, H. D. Taghirad, On the control of the kntu cdrpm: A cable driven redundant parallel manipulator, in: Intelligent Robots and Systems, 2008. IROS 2008. IEEE/RSJ International Conference on, IEEE, 2008, pp. 2404-2409.

[23] E. Ottaviano, A system for tension monitoring in cable-based parallel architectures, in: Proceedings of the 12th IFToMM World Congress, Besançon, France, Vol. 6, 2007.

[24] P. Bosscher, R. L. Williams, L. S. Bryson, D. Castro-Lacouture, Cablesuspended robotic contour crafting system, Automation in Construction 17 (1) (2007) $45-55$.

450 [25] A. Trevisani, Underconstrained planar cable-direct-driven robots: A trajectory planning method ensuring positive and bounded cable tensions, Mechatronics $20(1)(2010) 113-127$.

[26] G. Castelli, E. Ottaviano, P. Rea, A cartesian cable-suspended robot for improving end-users' mobility in an urban environment, Robotics and Computer-Integrated Manufacturing 30 (3) (2014) 335 343. doi:http: //dx.doi.org/10.1016/j.rcim.2013.11.001.

[27] G. Figliolini, P. Rea, Effects of the design parameters on the synthesis of geneva mechanisms, Proceedings of the Institution of Mechanical Engineers, Part C: Journal of Mechanical Engineering Science.

[28] A. Gonzalez Rodriguez, R. Morales Herrera, V. Feliu Batlle, P. Pintado Sanjuan, Improving the mechanical design of new staircase wheelchair, Industrial Robot: An International Journal 34 (2) (2007) 110-115.

[29] A. Gonzalez-Rodrguez, E. Ottaviano, F. Castillo-Garca, P. Rea, A novel design to improve pose accuracy for cable robots, in: The 14th IFToMM World Congress, Taipei, 2015. doi : 10.6567/IFToMM. 14TH.WC.0S2.018.

[30] R. E. Roberson, R. Schwertassek, Dynamics of multibody systems, Vol. 18, Springer-Verlag Berlin, 1988. 
[31] A. B. Alp, S. K. Agrawal, Cable suspended robots: design, planning and control, in: Robotics and Automation, 2002. Proceedings. ICRA'02. IEEE International Conference on, Vol. 4, IEEE, 2002, pp. 4275-4280.

[32] P. Gallina, A. Rossi, R. L. Williams II, Planar cable-direct-driven robots, part ii: Dynamics and control, in: Proceedings of the ASME IDETC/CIE Mechanics and Robotics Conference, 2001.

[33] J. Du, S. Agrawal, Dynamic modeling of cable-driven parallel manipulators with distributed mass flexible cables, ASME. Jnl. Vib. Acoust. 137 (2). doi:10.1115/1.4029486.

[34] V. Schmidt, A. Pott, Implementing extended kinematics of a cable-driven parallel robot in real-time, in: Cable-Driven Parallel Robots, Springer, 2013, pp. 287-298. 